\title{
HCN (1-0) opacity of outflowing gas in Arp 220W
}

\author{
J. Z. Wang ${ }^{1,2}$, S. Liu ${ }^{3,4}$, Z-Y. Zhang ${ }^{5,6}$, and Y. Shi ${ }^{5,6}$ \\ ${ }^{1}$ Shanghai Astronomical Observatory, Chinese Academy of Sciences,80 Nandan Road, Shanghai, 200030, PR China \\ e-mail: jzwang@shao.ac.cn \\ 2 Key Laboratory of Radio Astronomy, Chinese Academy of Sciences, 10 Yuanhua Road, Nanjing, JiangSu 210033, PR China \\ 3 National Astronomical Observatories, Chinese Academy of Sciences, Beijing 100012, PR China \\ e-mail: liushu@nao.cas.cn \\ ${ }^{4}$ CAS Key Laboratory of FAST, National Astronomical Observatories, Chinese Academy of Sciences, Beijing 100012, PR China \\ 5 School of Astronomy and Space Science, Nanjing University, Nanjing, 210093, PR China \\ ${ }^{6}$ Key Laboratory of Modern Astronomy and Astrophysics (Nanjing University), Ministry of Education, Nanjing 210093, PR China
}

Received xx / accepted xx

\begin{abstract}
Context. We present our findings for the $\mathrm{HCN} / \mathrm{H}^{13} \mathrm{CN}$ 1-0 line ratio in the molecular outflow of Arp 220 west based on high-resolution ALMA data

Aims. Molecular gas masses in the outflowing gas of galaxies driven by active galactic nuclei (AGNs) or starbursts are important parameters for understanding the feedback of these latter two phenomena and star-formation quenching. The conversion factor of line luminosities to masses is related to the optical depth of the molecular lines.

Methods. Using $\mathrm{H}^{13} \mathrm{CN} 1-0$, the isotopic line of $\mathrm{HCN} 1-0$, to obtain the line ratio of $\mathrm{HCN} / \mathrm{H}^{13} \mathrm{CN} 1-0$ is an ideal way to derive the optical depth of HCN 1-0 in outflowing gas.

Results. With the nondetection of $\mathrm{H}^{13} \mathrm{CN}$ 1-0 in the outflowing gas, a $3 \sigma$ lower limit of $\mathrm{HCN} / \mathrm{H}^{13} \mathrm{CN}$ 1-0 line ratio is obtained, which is at least three times higher than that found in the whole of the whole system of Arp 220 . The high $\mathrm{HCN} / \mathrm{H}^{13} \mathrm{CN} 1-0$ line ratio indicates low opacity of HCN 1-0 in the outflowing gas, even though the upper limit of HCN 1-0 opacity obtained here is still not good enough to draw any robust conclusions if HCN 1-0 is optically thin. A lower conversion factor of HCN 1-0 luminosity to dense gas mass in the outflowing gas should be used than that used for the host galaxy of Arp 220.
\end{abstract}

Key words. opacity -galaxies: individual: Arp 220 - methods: data analysis

\section{Introduction}

Mass outflows, a major manifestation of the feedback from active galactic nuclei (AGNs) and circumnuclear extreme starbursts (SBs), is one key element for understanding galaxy evolution (Feruglio et al. 2010; Sturm et al. 2011). Massive molecular outflows driven by AGNs or SBs have been found in galaxies with CO emissions (Feruglio et al. 2010; Cicone et al. 2012, 2014; Fluetsch et al. 2019) and far infrared $\mathrm{OH}$ absorption lines (Fischer et al. 2010). Outflow rates of molecular gas in starburstdominated galaxies have been found to be comparable to or even higher than their star formation rates (Cicone et al. 2014; Fluetsch et al. 2019). Furthermore, it has been suggested that a high-mass outflow rate can effectively quench star formation, which may cause the host galaxy to quickly leave the starforming phase to become an early-type gas-poor red galaxy.

Such outflows can also be detected with dense gas tracers. Examples are $\mathrm{HCN}, \mathrm{HCO}^{+}$, and $\mathrm{HNC}$ lines in Mrk 231 (Aalto et al. 2012, 2015), HCN 1-0 in Arp 220 (Barcos-Muñoz et al. 2018), and $\mathrm{HCO}^{+}$and $\mathrm{HCN}$ 1-0 in NGC 3256 (Michiyama et al. 2018). The mass-loss rate and outflow mass can be estimated with line luminosities of $\mathrm{HCN}$ and $\mathrm{HCO}^{+}$wings. However, such estimations strongly depend on the conversion factor from line luminosity to dense gas mass, which is related to the relative abundance of $\mathrm{HCN}$ and $\mathrm{HCO}^{+}$to hydrogen and the line opacity. The conversion factor of $\alpha_{H C N(1-0)}=3.3 M_{\odot}\left(\mathrm{K} \mathrm{km} \mathrm{s}^{-1} \mathrm{pc}^{2}\right)^{-1}$ whilst the optically thick assumption has been used to estimate the upper limit of the mass of the dense molecular outflow in Mrk 231 (Aalto et al. 2015), while $\alpha_{H C N(1-0)}$ is $0.24 M_{\odot}(\mathrm{K}$ $\left.\mathrm{km} \mathrm{s}^{-1} \mathrm{pc}^{2}\right)^{-1}$, if the optically thin assumption is made (BarcosMuñoz et al. 2018). On the other hand, Michiyama et al. (2018) used 0.24 and $10 M_{\odot}\left(\mathrm{K} \mathrm{km} \mathrm{s}^{-1} \mathrm{pc}^{2}\right)^{-1}$ to calculate the lower and upper limit of dense gas mass in NGC 3256. Isotopologue lines of $\mathrm{HCN}$ and $\mathrm{HCO}^{+}$molecules, such as $\mathrm{H}^{13} \mathrm{CN}$ and $\mathrm{H}^{13} \mathrm{CO}^{+}$ lines, can be used to determine the opacities of $\mathrm{HCN}$ and $\mathrm{HCO}^{+}$ lines with line ratios of $\mathrm{HCN} / \mathrm{H}^{13} \mathrm{CN}$ and $\mathrm{HCO}^{+} / \mathrm{H}^{13} \mathrm{CO}^{+}$, and were adopted to obtain averaged $\mathrm{HCN}$ opacities in nearby galaxies (Wang et al. 2016; Li et al. 2020).

Arp 220, as the nearest ultraluminous infrared galaxy (ULIRG) in the late stages of a galaxy merger, with strong outflowing gas at about $\pm 400 \mathrm{~km} \mathrm{~s}^{-1}$ and more than $10^{7} M_{\odot}$ mass around the western nucleus (Arp 220W) detected with HCN 1-0 (Barcos-Muñoz et al. 2018), is a good candidate to determine the line ratio of $\mathrm{HCN} / \mathrm{H}^{13} \mathrm{CN} 1-0$ for the outflowing gas, which can be used to determine the opacity of HCN 1-0 in outflowing gas.

In this letter, we describe the data from the ALMA archive. First we present our data reduction in $\$ 2$, before presenting our main results in $\$ 3$, and discussions in $\S 4$. We provide a brief summary in $\$ 5$. 


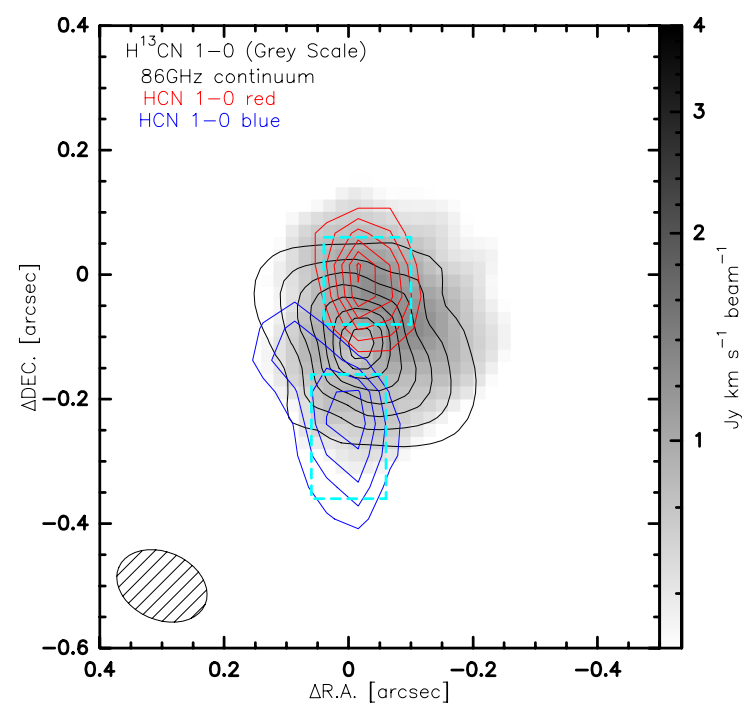

Fig. 1: The $86 \mathrm{GHz}$ continuum (black contour with levels starting from $3 \mathrm{mJy} \mathrm{beam}^{-1}$ in steps of $1 \mathrm{mJy}^{\text {beam }}{ }^{-1}$ ), a velocity integrated map of $\mathrm{H}^{13} \mathrm{CN} 1-0$ (grey scale, in units of $\mathrm{Jy} \mathrm{km} \mathrm{s}^{-1}$ beam ${ }^{-1}$ ), a velocity integrated map of HCN 1-0 red wing (red contour with levels starting from $0.2 \mathrm{Jy} \mathrm{km} \mathrm{s}^{-1}$ beam ${ }^{-1}$ in steps of $0.05 \mathrm{Jy} \mathrm{km} \mathrm{s}^{-1}$ beam $^{-1}$ ) and a velocity integrated map of HCN 1-0 blue wing (blue contour with levels starting from $0.2 \mathrm{Jy} \mathrm{km}$ $\mathrm{s}^{-1}$ beam ${ }^{-1}$ in steps of $0.05 \mathrm{Jy} \mathrm{km} \mathrm{s}^{-1}$ beam $^{-1}$ ), around the centre of Arp 220 west. The dashed cyan boxes are the regions for the spectra in Figures 2 and 3. The restoring beam $\left(0.151^{\prime \prime} \times 0.109\right.$ ", $\left.P A=23.4^{\circ}\right)$ of the data cube is shown in the bottom left. The central coordinates of this map are R.A.: 15:34:57.22 and Dec: 23:30:11.6 (J2000).

\section{The data}

The data were taken from the ALMA archival system (project number: 2015.1.00702.S, PI: L. Barcos-Muñoz), the same data set used in Barcos-Muñoz et al. (2018). Standard bandpass, phase, and flux calibrations using scripts from the archive system were done in CASA, as well as imaging and deconvolution using natural weighting with a pixel size of $0.02^{\prime \prime}$ and frequency resolution of $3.906 \mathrm{MHz}$ in the task 'tclean'. The continuum image obtained from line-free channels, the velocity-integrated maps of HCN 1-0 at the rest frequency of $88.6318473 \mathrm{GHz}$ for both red and blue wings, as well as the velocity integrated maps of the optically thin isotopologue $\mathrm{H}^{13} \mathrm{CN} 1-0$, were obtained from the cleaned datacube (see Figure 1). Spectra of $\mathrm{HCN}$ and $\mathrm{H}^{13} \mathrm{CN} 1-$ 0 at the rest frequency of $86.3401764 \mathrm{GHz}$ toward red and blue wings from the regions marked as dashed cyan boxes in Figure 1 were also obtained and are presented in Figures 2 and 3.

\section{Results}

\subsection{Spatial distribution of outflow traced by HCN}

The velocity-integrated flux distribution of the red and blue wings of HCN 1-0, as well as the continuum emission around $86 \mathrm{GHz}$ and the velocity-integrated flux of $\mathrm{H}^{13} \mathrm{CN} 1-0$ near the centre of Arp 220W are presented in Figure 1. Outflows near the centre of Arp 220W can be seen with HCN 1-0 emission, which is consistent with the findings reported by Barcos-Muñoz et al. (2018), with a blue wing at the northern side and a red wing at the southern side of the centre of Arp 220W traced by continuum emission. As the abundance of $\mathrm{H}^{13} \mathrm{CN}$ is less than one-

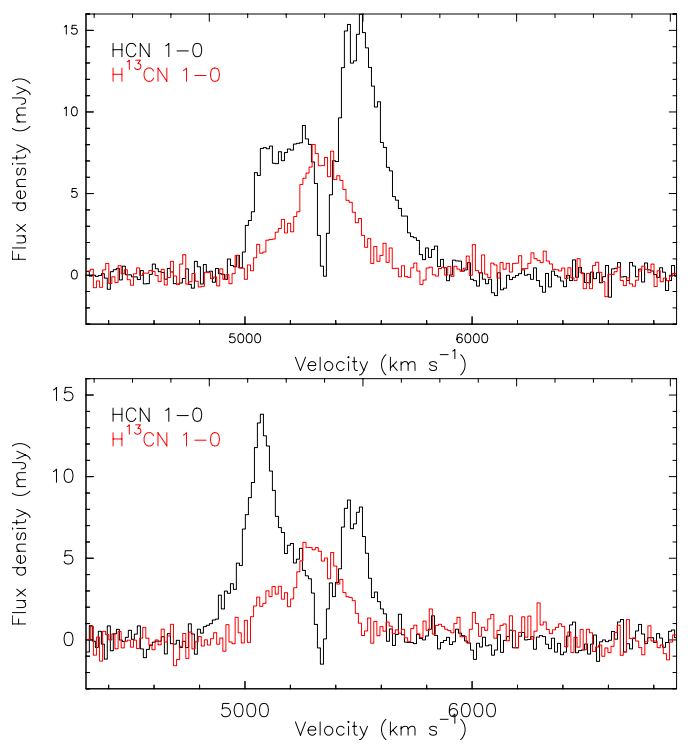

Fig. 2: Top: $\mathrm{HCN}$ (black) and $\mathrm{H}^{13} \mathrm{CN} 1-0$ (red) spectra collected from the red wing region of the cyan box in Figure 1. Bottom: Same as the top spectra but from the blue wing region. Radiodefined velocities are used, while the system velocity is about $5350 \mathrm{~km} \mathrm{~s}^{-1}$.

tenth of $\mathrm{HCN}, \mathrm{H}^{13} \mathrm{CN}$ 1-0 is normally optically thin in Galactic star forming regions (Padovani et al. 2011; Colzi et al. 2018) and galaxies (Henkel et al. 1998; Li et al. 2020). However, $\mathrm{H}^{13} \mathrm{CN} 1-$ 0 in Arp 220W shows an offset of the peak emission from that of the continuum, obtained from (see Figure 1). Such an offset may be caused by absorption of $\mathrm{H}^{13} \mathrm{CN}$ 1-0 towards continuum emission around the centre region, where the absorption of $\mathrm{HCN}$ 1-0 is significantly below the continuum level at some velocity ranges. HCN 1-0 absorption can be important even at the positions with less continuum emission than the centre (see Figure 2 and 3 ).

Collecting the most significant emitting regions of red and blue wings marked as dashed cyan boxes in Figure 1, HCN and $\mathrm{H}^{13} \mathrm{CN} 1-0$ spectra are obtained to determine the line ratios of $\mathrm{HCN} / \mathrm{H}^{13} \mathrm{CN} 1-0$ in the line wings. The red wing displayed in Figure 1 shown with red contours is integrated from 5620 to $5800 \mathrm{~km} \mathrm{~s}^{-1}$ as radio-defined velocity, while the blue wing shown with blue contours is integrated from 4860 to $5040 \mathrm{~km}$ $\mathrm{s}^{-1}$. The SO line at the rest frequency of $86.093983 \mathrm{GHz}$ and $\mathrm{HC}^{15} \mathrm{~N} 1-0$ at the rest frequency of $86.054967 \mathrm{GHz}$ can contaminate the red wing of $\mathrm{H}^{13} \mathrm{CN} 1-0$ (see Figure 3), especially with velocity higher than $6000 \mathrm{~km} \mathrm{~s}^{-1}$. Therefore, in order to avoid such contaminations when estimating the $\mathrm{HCN} / \mathrm{H}^{13} \mathrm{CN} 1-0$ ratio, even though there are no such contaminations for the HCN 1-0 line, only the velocity range between 5620 and $5800 \mathrm{~km} \mathrm{~s}^{-1}$ is integrated for the red wing.

\section{2. $\mathrm{HCN} / \mathrm{H}^{13} \mathrm{CN}$ 1-0 line ratio}

The HCN 1-0 emission is no more than five times the corresponding $\mathrm{H}^{13} \mathrm{CN} 1-0$ emission in most of the velocity components towards the red and blue wings of the outflowing gas (see Figure 3). The $\mathrm{HCN} / \mathrm{H}^{13} \mathrm{CN}$ 1-0 ratio in the entire system of Arp 220 is about five according to single-dish observations with IRAM 30-meter telescope (Wang et al. 2016). HCN and $\mathrm{H}^{13} \mathrm{CN}$ 1-0 fluxes measured from ALMA data are consistent with those obtained with the IRAM 30-meter telescope (Wang 
et al. 2016). $\mathrm{HCN} / \mathrm{H}^{13} \mathrm{CN}$ 1-0 ratios towards the outflowing regions vary along with different velocities (see Figure 2 and 3 ), from more than five to even less than one at the line centre, which seems likely to be caused by the absorption of HCN 1-0 towards continuum emission or the self absorption of HCN 1-0 with a temperature gradient. Such an absorption, where the velocity of the HCN 1-0 absorption peak is similar to that of the $\mathrm{H}^{13} \mathrm{CN}$ 1-0 emission peak, will cause an overestimation of opacity if a unique temperature is assumed for the molecular gas. However, such absorption is mainly caused by gas from the disc instead of the outflowing gas, based on the velocity information.

The line profile of HCN 1-0 from the red wing region (see Figure 3 ) in the blue part from about 4950 to $5100 \mathrm{~km} \mathrm{~s}^{-1}$ agrees well with the spectra of $\mathrm{H}^{13} \mathrm{CN}$ 1-0 from the same region, with a line ratio of about five. On the other hand, the intensity of $\mathrm{HCN}$ 1-0 is well above five times that of $\mathrm{H}^{13} \mathrm{CN} \mathrm{1-0}$ at the red part, especially from 5620 to $5800 \mathrm{~km} \mathrm{~s}^{-1}$. The SO line at the rest frequency of $86.093983 \mathrm{GHz}$ contaminates $\mathrm{H}^{13} \mathrm{CN} 1-0$ from $\sim 5850$ $\mathrm{km} \mathrm{s}^{-1}$ to $6100 \mathrm{~km} \mathrm{~s}^{-1}$. Thus, it is hard to obtain a reliable ratio of $\mathrm{HCN}$ and $\mathrm{H}^{13} \mathrm{CN}$ 1-0 at velocity ranges with SO line contamination. SO and $\mathrm{HC}^{15} \mathrm{~N}$ 1-0 emission can also be seen in the blue wing regions (See Figure 3). However, the line profiles of HCN and $\mathrm{H}^{13} \mathrm{CN}$ 1-0 from the blue wing region agree well with each other in the red part without SO contamination, with a line ratio also similar to five, while HCN 1-0 intensity is well above five times that of $\mathrm{H}^{13} \mathrm{CN}$ 1-0 in the blue region (see Figure 3). In summary, $\mathrm{HCN} 1-0$ emission is significantly above five times that of $\mathrm{H}^{13} \mathrm{CN} 1-0$ from the outflowing gas, while it is equal to or below five times that of the $\mathrm{H}^{13} \mathrm{CN} 1-0$ emission from the gas that is not outflowing.

The HCN 1-0 velocity-integrated flux of the red wing in Figures 2 and 3 between 5620 and $5800 \mathrm{~km} \mathrm{~s}^{-1}$ is $631.1 \pm 22.5 \mathrm{mJy}$ $\mathrm{km} \mathrm{s}^{-1}$, while this value is $794.1 \pm 27.7 \mathrm{mJy} \mathrm{km} \mathrm{s}^{-1}$ for the blue wing between 4860 and $5040 \mathrm{~km} \mathrm{~s}^{-1}$ (see Table 1). The uncertainties are estimated with $\sigma_{r m s} \times \sqrt{\delta v \Delta V}$, where $\sigma_{r m s}$ is the rms with baseline fitting for the spectra shown in Figure 2 at the velocity resolution of $\delta v$ as $\sim 13.2 \mathrm{~km} \mathrm{~s}^{-1}$, while $\Delta V$ is $180 \mathrm{~km} \mathrm{~s}^{-1}$ which is the integrated line width for both red and blue wings. The velocity-integrated fluxes of $\mathrm{H}^{13} \mathrm{CN} \mathrm{1-0}$ at the corresponding velocities are 59.7 and $61.5 \mathrm{mJy} \mathrm{km} \mathrm{s}^{-1}$ for the red and blue wings, respectively, which is below the $3 \sigma$ level for both components. The noise level near $\mathrm{H}^{13} \mathrm{CN} 1-0$ is about 0.94 of that of the HCN 1-0 line. Thus, the $3 \sigma$ values 63.2 and $77.9 \mathrm{mJy} \mathrm{km} \mathrm{s}^{-1}$ are used for the red and blue wings, respectively, which give line ratios of $\mathrm{HCN} / \mathrm{H}^{13} \mathrm{CN} 1-0$ greater than 9.5 and 9.7 in the red and blue wings, after converting the units from mJy for flux density to $\mathrm{mK}$ for brightness temperature.

The line ratio $\mathrm{HCN} / \mathrm{H}^{13} \mathrm{CN}$ 1-0 varies from $7.6 \pm 2.8$ in $\mathrm{NGC}$ 4418 to $40 \pm 3.6$ in M82 within a sample of local galaxies (Li et al. 2020). Thus, the high $\mathrm{HCN} / \mathrm{H}^{13} \mathrm{CN} 1-0$ ratio of greater than 9.6 in the line wings of Arp $220 \mathrm{~W}$ is not extremely high in galaxies. On the other hand, the $\mathrm{HCN} / \mathrm{H}^{13} \mathrm{CN}$ 1-0 line ratio of about five for the whole galaxy of Arp 220 (Wang et al. 2016) indicates that HCN column density in Arp 220 is extremely high, which is consistent with our knowledge of Arp 220 as a gas-rich latestage merger with extreme starburst activity.

\section{Discussion}

\subsection{HCN 1-0 opacity in the outflowing gas}

The average line ratio of $\mathrm{HCN} / \mathrm{H}^{13} \mathrm{CN} 1-0$ in Arp 220 is about five based on single-dish observations (Wang et al. 2016), which gives an average opacity of $\sim 22$ for $\mathrm{HCN} 1-0$, assuming the

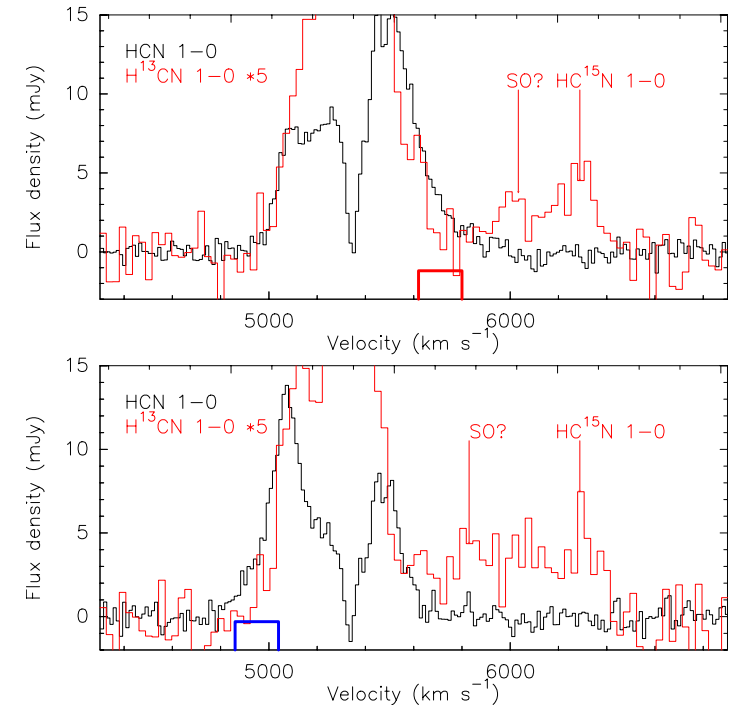

Fig. 3: As in Figure 2, but with $\mathrm{H}^{13} \mathrm{CN} 1-0$ multiplied by 5. The red window in the top panel is for the velocity range from 5620 to $5800 \mathrm{~km} \mathrm{~s}^{-1}$ which integrates the red wing of the outflow in Figure 1, while the blue window in the bottom panel is for the blue wing from 4860 to $5040 \mathrm{~km} \mathrm{~s}^{-1}$.

abundance ratio $\left(X_{H C N} / X_{H^{13} C N}\right)$ is the same as the ${ }^{12} \mathrm{C} /{ }^{13} \mathrm{C}$ ratio, namely 100 , which was suggested by Martín et al. (2011) based on observations of ${ }^{13} \mathrm{CO} / \mathrm{C}^{18} \mathrm{O} 2-1$. The optical depth is obtained with $\frac{\mathrm{I}_{\mathrm{H}^{13} \mathrm{CN} 1-0}}{\mathrm{I}_{\mathrm{HCN} 1-0}}=\frac{1-e^{-\tau\left(\mathrm{H}^{13} \mathrm{CN} 1-0\right)}}{1-e^{-\tau(\mathrm{HCN} 1-0)}}$, where $\frac{\mathrm{I}_{\mathrm{H}^{13} \mathrm{CN} 1-0}}{\mathrm{I}_{\mathrm{HCN} 1-0}}$ is the measured line ratio, while $\frac{\tau\left(\mathrm{H}^{13} \mathrm{CN} 1-0\right)}{\tau(\mathrm{HCN} 1-0)}$ is assumed to be the same as the abundance ratio of ${ }^{13} \mathrm{C} /{ }^{12} \mathrm{C}$. By adopting a $\mathrm{HCN} / \mathrm{H}^{13} \mathrm{CN} 1-0$ ratio of five, the line profile of $\mathrm{H}^{13} \mathrm{CN}$ is close to that of $\mathrm{HCN}$ in the velocity ranges of $5000-5100 \mathrm{~km} \mathrm{~s}^{-1}$ (Figure 3 top) and $5500-5600 \mathrm{~km} \mathrm{~s}^{-1}$ (Figure 3 bottom). This illustrates that there are no strong contaminations from HCN 1-0 absorption in the two velocity ranges, and that these ranges are not affected by outflowing gas in the regions with red and blue wings near Arp $220 \mathrm{~W}$. For the emissions near the central regions of both Arp 220 west and east, absorption of both $\mathrm{HCN} 1-0$ and $\mathrm{H}^{13} \mathrm{CN} 1-0$ cannot be neglected, which poses a problem for estimating opacity of HCN 1-0 there. Absorption of HCN 1-0 towards the continuum emission or HCN 1-0 emission from inner warm dense molecular gas can result in overestimation of the opacity of $\mathrm{HCN}$ 1-0 because of the underestimation of HCN 1-0 emission.

The line ratios of $\mathrm{HCN} / \mathrm{H}^{13} \mathrm{CN} 1-0$ in the red and blue wings are greater than 9.5 and 9.7, respectively, giving a $3 \sigma$ upper limit of $\sim 0.1$ for the opacity of $\mathrm{H}^{13} \mathrm{CN} 1-0$ in the outflowing gas. Assuming an $X_{H C N} / X_{H^{13} C N}$ abundance ratio of 100 as suggested by Martín et al. (2011) with the ${ }^{13} \mathrm{CO} / \mathrm{C}^{18} \mathrm{O}$ 2-1 line ratio obtained with the Submillimeter Array (SMA), the opacity of HCN 1-0 should be less than 10.5 .

The HCN 1-0 flux combined between 5620 and $5800 \mathrm{~km}$ $\mathrm{s}^{-1}$ in the red wing and between 4860 and $5040 \mathrm{~km} \mathrm{~s}^{-1}$ in the blue wing integrated within the dashed cyan boxes in Figure 1 is $1425.2 \pm 35.7 \mathrm{mJy} \mathrm{km} \mathrm{s}^{-1}$, where the flux is from the sum of HCN 1-0 flux from the red wing of $631.1 \mathrm{mJy} \mathrm{km} \mathrm{s}^{-1}$, and that from the blue wing of $794.1 \mathrm{mJy} \mathrm{km} \mathrm{s}^{-1}$, while the noise level is $\sqrt{22.5^{2}+27.7^{2}} \mathrm{mJy} \mathrm{km} \mathrm{s}{ }^{-1}$. The corresponding $\mathrm{H}^{13} \mathrm{CN} 1-0$ flux is $121.3 \pm 35.7 \mathrm{mJy} \mathrm{km} \mathrm{s}^{-1}$ derived in the same way, that is, $59.7+61.5 \mathrm{mJy} \mathrm{km} \mathrm{s}{ }^{-1}$ with noise of $\sqrt{21.1^{2}+25.9^{2}} \mathrm{mJy} \mathrm{km}$ $\mathrm{s}^{-1}$. In other words, $\mathrm{H}^{13} \mathrm{CN} 1-0$ emission is about $3.4 \sigma$. How- 
Table 1: HCN 1-0 and $\mathrm{H}^{13} \mathrm{CN} 1-0$ in the outflows of Arp 220W

\begin{tabular}{ccc}
\hline \hline Line & $\begin{array}{c}\text { Integrated flux } \\
\mathrm{mJy} \mathrm{km} \mathrm{s}^{-1}\end{array}$ & Optical depth \\
\hline HCN 1-0 (blue) & $794.1 \pm 27.7$ & $<4.4$ \\
$\mathrm{H}^{13} \mathrm{CN} \mathrm{1-0} \mathrm{(blue)}$ & $<83.9(3 \sigma)$ & $<0.11$ \\
$\mathrm{HCN} \mathrm{1-0} \mathrm{(red)}$ & $631.1 \pm 22.5$ & $<4.4$ \\
$\mathrm{H}^{13} \mathrm{CN} \mathrm{1-0} \mathrm{(red)}$ & $<67.5(3 \sigma)$ & $<0.11$ \\
\hline
\end{tabular}

Note. The optical depth of HCN 1-0 is estimated with a ${ }^{12} \mathrm{C} /{ }^{13} \mathrm{C}$ ratio of 40 (Wheeler et al. 2020).

ever, as the low-velocity components are included, which may be mainly from the disc instead of outflowing gas, the $\mathrm{H}^{13} \mathrm{CN}$ 1-0 emission contribution should be mainly from optically thick non-outflowing gas. Thus, the non-detection of $\mathrm{H}^{13} \mathrm{CN} 1-0$ emission from the outflowing gas should still be considered and a $3 \sigma$ upper limit should be used for estimating the line ratio of $\mathrm{HCN} / \mathrm{H}^{13} \mathrm{CN} 1-0$ for the combined regions of outflowing gas, which gives a ratio of greater than 13.3 and an opacity of $\mathrm{H}^{13} \mathrm{CN} 1-0$ of less than 0.078 , or less than 0.11 for both the red and blue wings if calculating them individually. Assuming the $\mathrm{HCN} / \mathrm{H}^{13} \mathrm{CN}$ abundance ratio of 100 as suggested by Martín et al. (2011), the opacity of HCN 1-0 should be less than 7.8, which is about one-third of the average value in Arp 220. Thus, even though we do not have enough information to derive the opacity of HCN 1-0 in the outflowing gas, the upper limit on HCN 1-0 opacity in the outflowing gas is less than one-third of the average value in Arp 220.

The ${ }^{12} \mathrm{C} /{ }^{13} \mathrm{C}$ ratio in Arp 220 was updated to be 40 with ALMA observations of ${ }^{12} \mathrm{CO} 3-2$ and ${ }^{13} \mathrm{CO} 4-3$ (Wheeler et al. 2020). Therefore, the opacity of HCN 1-0 should be about $40 \%$ of the opacity estimated using the ${ }^{12} \mathrm{C} /{ }^{13} \mathrm{C}$ ratio of 100 suggested by Martín et al. (2011), both of which are less than 4.4 as listed in Table 1 . Even with a $3 \sigma$ upper limit of HCN 1-0 opacity in the outflowing gas combined for the red and blue components of 3.1 , it is still not possible to draw a firm conclusion as to whether HCN 1-0 is optically thin or thick in the outflowing gas of Arp 220. The average opacity of HCN 1-0 in the whole galaxy is down to $\sim 9$ using the updated ${ }^{12} \mathrm{C} /{ }^{13} \mathrm{C}$ in Arp 220 (Wheeler et al. 2020). The optical depth of HCN 1-0 in the outflowing gas is at least three times less than that in the host galaxy of Arp 220 if the same $\mathrm{HCN} / \mathrm{H}^{13} \mathrm{CN}$ abundance ratio is used. However, the possibility of higher $\mathrm{HCN} / \mathrm{H}^{13} \mathrm{CN}$ abundance ratio in the outflowing gas than that in the galaxy can also enhance the $\mathrm{HCN} / \mathrm{H}^{13} \mathrm{CN} 1-0$ line ratio, even though it is not likely to lead to significantly different line ratios between the outflowing gas and the gas in the whole galaxy. In summary, even though we can conclude that the optical depth of HCN 1-0 in the outflowing gas is at least a factor of three lower than the average value for the whole galaxy, whether HCN 1-0 is optically thick or thin remains uncertain.

\subsection{Outflow properties and future prospects}

Therefore, even though the accurate opacity of HCN 1-0 of the outflowing gas in Arp 220W cannot be determined because of the limited sensitivity of the observations, the non-detection of $\mathrm{H}^{13} \mathrm{CN} 1-0$ from the outflowing gas provides a good lower limit on the $\mathrm{HCN} / \mathrm{H}^{13} \mathrm{CN}$ 1-0 line ratio there. Based on this ratio, an upper limit on the HCN 1-0 opacity of less than one-third of the average value over the whole of Arp 220 can be obtained. It is necessary to use a smaller conversion factor of HCN 1-0 luminosity to dense gas mass than that used for normal galaxies, namely of $10 M_{\odot}\left(\mathrm{K} \mathrm{km} \mathrm{s}^{-1} \mathrm{pc}^{2}\right)^{-1}$ (Michiyama et al. 2018). The conversion factor under optically thin conditions, namely 0.24 $M_{\odot}\left(\mathrm{K} \mathrm{km} \mathrm{s}^{-1} \mathrm{pc}^{2}\right)^{-1}$, was used to estimate the lower limit of outflowing mass and mass loss rate in Arp 220W (Barcos-Muñoz et al. 2018). The CO 1-0 luminosity of the outflowing gas in Arp 220W is similar to that of HCN 1-0 (Barcos-Muñoz et al. 2018), which means an even higher $\mathrm{HCN} / \mathrm{CO} 1-0$ luminosity ratio than that in the massive outflowing gas from the central AGN in Mrk 231 (Aalto et al. 2012). If the same conversion factor of line luminosity to mass ratio is used in Arp 220W and Mrk 231, the dense gas fraction in the outflowing gas in Arp 220W is higher than that in Mrk 231, and even the outflowing gas in Mrk 231 is more massive than that in Arp 220W.

However, such conversion factors strongly depend on the opacity of $\mathrm{CO}$ and $\mathrm{HCN}$ lines, as well as HCN and $\mathrm{CO}$ abundances in the outflowing gas. The abundance issue of $\mathrm{HCN}$ when determining dense gas, which can cause uncertainties of estimating the dense gas mass, can be done with lines of different molecules with high dipole moments, such as $\mathrm{HCN}, \mathrm{HCO}^{+}$, HNC, and CS. With observations of different lines of similar critical density, the effect of chemical enhancement of special molecules, such as $\mathrm{HCN}$ or $\mathrm{HCO}^{+}$, can be found, as discussed for the outflowing gas in Mrk 231 (Lindberg et al. 2016). The outflowing gas traced by $\mathrm{HCO}^{+} 1-0$ shows similar distribution to that of HCN 1-0 in Arp 220W, with the same dataset (BarcosMunoz 2016), which indicates that astrochemical enhancement of the abundance of the HCN molecule is not significant in the outflowing gas. However, new observations of HNC 1-0 and CS 2-1 are required to further confirm such abundance effects.

The opacity of lines in the outflowing gas can be determined from observations of their optically thin isotopologues, such as $\mathrm{H}^{13} \mathrm{CN} 1-0$ for $\mathrm{HCN}$ 1-0. The data used in this work were obtained from observations lasting about 1.9 hours on source with ALMA. If $\mathrm{H}^{13} \mathrm{CN}$ 1-0 data of three times higher sensitivity are required to obtain the $3 \sigma$ upper limit of $\mathrm{HCN} 1-0$ opacity of about 1 with a ${ }^{12} \mathrm{C} /{ }^{13} \mathrm{C}$ ratio of approximately 40 measured with ALMA (Wheeler et al. 2020), the required total telescope time would be about 20 hours; including overheads, this would be prohibitively expensive. Using the RADEX online calculator (van der Tak et al. 2007), when HCN molecules are close to local thermal equilibrium (LTE), the opacity of HCN 2-1 is about 3.5 times that of HCN 1-0 under optically thin conditions. Therefore, it would be more effective to derive the opacity of $\mathrm{HCN}$ 2-1 with observations of $\mathrm{HCN}$ and $\mathrm{H}^{13} \mathrm{CN}$ 2-1 than that of $\mathrm{HCN}$ $1-0$. $\mathrm{H}^{13} \mathrm{CN} 2-1$ in the outflowing gas in Arp $220 \mathrm{~W}$ can be expected to be detected within several hours using ALMA, because HCN 2-1 has moderate opacity of around 0.5 to 1.0, even though HCN 1-0 is optically thin. Otherwise, an upper limit on HCN 2-1 opacity of approximately 0.5 would also be useful to determine whether or not HCN 1-0 is optically thin. Stronger HCN 2-1 emission than that of 1-0 was also found in the outflowing gas of Mrk 231 (Lindberg et al. 2016) without significant line contamination of HCN 2-1. On the other hand, there is one strong line within $\pm 700 \mathrm{~km} \mathrm{~s}^{-1}$ range of $\mathrm{H}^{13} \mathrm{CN} 2-1$, namely $\mathrm{HC}_{3} \mathrm{~N} 19-18$ at $172.849287 \mathrm{GHz}$ at a velocity of $\sim-300 \mathrm{~km} \mathrm{~s}^{-1}$ relative to $\mathrm{H}^{13} \mathrm{CN}$ 2-1 at $172.67796 \mathrm{GHz}$. Because of the relatively low abundance ratio of $\mathrm{HC}_{3} \mathrm{~N}$ to $\mathrm{HCN}$ and the high density and temperature requirement for the excitation of $J=19$, contamination of $\mathrm{HC}_{3} \mathrm{~N}$ $19-18$ at the outflowing region can be neglected.

Deep ${ }^{13} \mathrm{CO} 2-1$ and 1-0 observations may also be useful for determining the opacities of CO 1-0 and 2-1 in Arp 220W. However, because the line luminosity of $\mathrm{CO} 1-0$ is comparable to that of HCN 1-0, there is no great advantage to using ${ }^{13} \mathrm{CO}$ lines instead of $\mathrm{H}^{13} \mathrm{CN}$. Combining ${ }^{13} \mathrm{CO} 2-1$ and $\mathrm{CO} 2-1$ data with those for $\mathrm{HCN} 2-1$ and $\mathrm{H}^{13} \mathrm{CN}$ 2-1 may be a good choice for de- 
termining whether $\mathrm{CO}$ and $\mathrm{HCN}$ lines in the outflowing gas are optically thick or thin. $\mathrm{H}^{13} \mathrm{CN}$ or ${ }^{13} \mathrm{CO}$ lines towards local massive AGN molecular outflows or other extreme starbursts with strong outflow, such as NGC 3256 (Michiyama et al. 2018) or M82 (Chisholm \& Matsushita 2016), will be helpful for understanding such outflows.

\section{Summary}

We present an ideal method to derive optical depths of HCN 10 in Arp 220 using the $\mathrm{HCN} / \mathrm{H}^{13} \mathrm{CN}$ line ratios. We apply this method to the outflowing gas of galaxies and use it to better determine the conversion factor from line luminosity to mass. With information from spatially resolved $\mathrm{H}^{13} \mathrm{CN} 1-0$ and $\mathrm{HCN} 1-0$ observations in Arp 220 with ALMA, we obtain a $3 \sigma$ lower limit on the $\mathrm{HCN} / \mathrm{H}^{13} \mathrm{CN} 1-0$ line ratio in the outflowing gas of Arp $220 \mathrm{~W}$ that is at least three times that found for the whole system of Arp 220. Such a line ratio indicates that the opacity of HCN 1-0 in the outflowing gas of Arp 220W is at least several times lower than that in other regions of Arp 220. Therefore, a lower conversion factor of HCN 1-0 luminosity to dense gas mass should be used in the outflowing gas than that used for the whole galaxy of Arp 220. Further sensitive observations of $\mathrm{HCN} / \mathrm{H}^{13} \mathrm{CN} 2-1$ or 1-0 with ALMA are necessary to constrain the opacity of HCN lines in the outflowing gas in Arp 220W.

Acknowledgements. We thank the anonymous referee for helpful comments to improve the manuscript. This work is supported by the National Natural Science Foundation of China grant 11988101, 11590783, and U1731237. This paper makes use of the following ALMA data: ADS/JAO.ALMA\#2015.1.00702.S. ALMA is a partnership of ESO (representing its member states), NSF (USA) and NINS (Japan), together with NRC (Canada), MOST and ASIAA (Taiwan), and KASI (Republic of Korea), in cooperation with the Republic of Chile. The Joint ALMA Observatory is operated by ESO, AUI/NRAO and NAOJ.

\section{References}

Aalto, S., Garcia-Burillo, S., Muller, S., et al. 2012, A\&A, 537, A44. doi:10.1051/0004-6361/201117919

Aalto, S., Garcia-Burillo, S., Muller, S., et al. 2015, A\&A, 574, A85. doi:10.1051/0004-6361/201423987

Barcos-Munoz, L. 2016, Ph.D. Thesis. doi:10.18130/V31S5F

Barcos-Muñoz, L., Aalto, S., Thompson, T. A., et al. 2018, ApJ, 853, L28. doi:10.3847/2041-8213/aaa28d

Chisholm, J. \& Matsushita, S. 2016, ApJ, 830, 72. doi:10.3847/0004$637 \mathrm{X} / 830 / 2 / 72$

Cicone, C., Feruglio, C., Maiolino, R., et al. 2012, A\&A, 543, A99. doi:10.1051/0004-6361/201218793

Cicone, C., Maiolino, R., Sturm, E., et al. 2014, A\&A, 562, A21. doi:10.1051/0004-6361/201322464

Colzi, L., Fontani, F., Caselli, P., et al. 2018, A\&A, 609, A129. doi:10.1051/0004-6361/201730576

Feruglio, C., Maiolino, R., Piconcelli, E., et al. 2010, A\&A, 518, L155. doi:10.1051/0004-6361/201015164

Fischer, J., Sturm, E., González-Alfonso, E., et al. 2010, A\&A, 518, L41. doi:10.1051/0004-6361/201014676

Fluetsch, A., Maiolino, R., Carniani, S., et al. 2019, MNRAS, 483, 4586. doi: $10.1093 / \mathrm{mnras} / \mathrm{sty} 3449$

Henkel, C., Asiri, H., Ao, Y., et al. 2014, A\&A, 565, A3. doi:10.1051/00046361/201322962

Henkel, C., Chin, Y.-N., Mauersberger, R., et al. 1998, A\&A, 329, 443

Li, F., Wang, J., Fang, M., et al. 2020, MNRAS, 494, 1095. doi:10.1093/mnras/staa676

Lindberg, J. E., Aalto, S., Muller, S., et al. 2016, A\&A, 587, A15. doi:10.1051/0004-6361/201527457

Martín, S., Krips, M., Martín-Pintado, J., et al. 2011, A\&A, 527, A36. doi:10.1051/0004-6361/201015855

Michiyama, T., Iono, D., Sliwa, K., et al. 2018, ApJ, 868, 95. doi:10.3847/15384357/aae82a

Padovani, M., Walmsley, C. M., Tafalla, M., et al. 2011, A\&A, 534, A77. doi:10.1051/0004-6361/201117134
Sturm, E., González-Alfonso, E., Veilleux, S., et al. 2011, ApJ, 733, L16. doi:10.1088/2041-8205/733/1/L16

van der Tak, F. F. S., Black, J. H., Schöier, F. L., et al. 2007, A\&A, 468, 627. doi:10.1051/0004-6361:20066820

Wang, J., Zhang, Z.-Y., Zhang, J., et al. 2016, MNRAS, 455, 3986. doi: $10.1093 /$ mnras/stv2580

Wheeler, J., Glenn, J., Rangwala, N., et al. 2020, ApJ, 896, 43. doi:10.3847/1538-4357/ab8f32 